\title{
Adsorption processes in solving environmental problems in water sources of Urban Territories Development
}

\author{
Tamara Krasnova ${ }^{1, *}$, Mikhail Kirsanov ${ }^{1}$, Oxana Belyaeva $^{1}$ and Irina Timoshchuk ${ }^{1}$ \\ ${ }^{1}$ Kemerovo State University, Kemerovo, 650000 Russia
}

\begin{abstract}
Currently, there is a high level of environmental pollution Urban Territories, which poses a danger to ecosystems and public health, since a significant part of the industrial wastewater generated is discharged into water bodies neither sufficiently purified no without purification. The existing methods for treating effluents containing low concentrations of organic substances are generally quite expensive, long, require significant amounts of reagents or energy-intensive, often accompanied by the formation of secondary pollution and the loss of valuable substances. The scientific significance of the research results lies in the development of the adsorption theory of different nature organic substances by carbon sorbents, which differ in structure and properties of the surface. The values of the adsorption parameters calculated for the investigated active coals calculated from the experimental data can be used in the database of the sorbents' main parameters. The practical importance lies in the choice and recommendations for the practical application of active coals with the best adsorption characteristics, methods for increasing the efficiency of organic components adsorption and obtaining equilibrium data for calculating process parameters of the wastewater treatment process
\end{abstract}

\section{Introduction}

Ensuring cost-effective and environmentally safe water use, protecting water resources from depletion, pollution and degradation is one of the most important tasks of our time. Currently, a high level of water resources pollution of the Russian Federation is achieved, due to the economic activity. Wastewater, chemical and pharmaceutical industries containing mainly toxic organic contaminants, including phenol, chlorophenol, chloroform, aldehydes, pyridin, aniline, nitrobenzene, caprolactam, represent a special danger for water bodies. To protect water resources from pollution, depletion and degradation, it is necessary to exclude wastewater discharge and reduce fresh water intake by creating environmentally safe production facilities with closed water cycles and the disposal of hazardous components. The solution of such problems is possible on the basis of fundamental science development, an integrated scientific and technical approach.

\footnotetext{
*Corresponding author: ecolog1528@yandex.ru
} 
The purpose of this work is to establish the regularities, features and mechanism of organic contaminants adsorption on carbon adsorbents with different properties for the creation of environmentally safe adsorption technologies.

A review of the water purification known methods from organic compounds of various classes allows us to conclude that, despite their diversity, there is no such universal and economical method that could be recommended for purification of any waters from organic compounds in a wide concentration range, with the isolation of valuable components from the purified water, there is a lack of information on the extraction of compounds number (Koganovskii, 1983; Kirsanov, 2007; Krasnova, 2011). The use of adsorption methods for purification of natural and waste water opens wide prospects in this direction (Koganovskii, 1990). Adsorption methods are characterized by high efficiency, the ability to purify water containing low concentrations of organic substances, up to MPC and below, the ability to allocate valuable products from water (Krasnova, 2011). Adsorption plants for water treatment attract attention with its compactness, simplicity of instrumentation and process control, low cost with a high degree of purification.

Phenol adsorption from individual solutions was adequately covered (Koganovskii, 1990; Gorelkina, A.K., 2006). At the same time, only isolated data are presented concerning the adsorption of chlorophenol, chloroform, aldehydes, pyridine, aniline, nitrobenzene, caprolactam from aqueous solutions with active carbons. There is no information about their behavior in a joint presence.

\section{Materials and Methods}

The objects of research were:

a) industrial granulated active coals (AC) based on coal-bearing raw materials of AGOV-1, CKD-515, AG-3, AG-5, brands (producer of the SPA Sorbent, Perm), and crushed BAU wood, producer of the SPA Sorbent, Perm), KsAU (coconut, producer of Eurocarb, UK) and ABG (brown coal semi-carbon, Karbonica, Krasnoyarsk), characterized by nature, production method, porous structure and chemical state of the surface ;

b) model aqueous solutions of individual components and mixtures of phenol, chloroform, n-chlorophenol, formaldehyde, acetaldehyde; nitrobenzene, $\varepsilon$-caprolactam, aniline, pyridine; all the reagents used corresponded to the required degree of purity.

All AC were preliminary washed by distilled water from dust fractions and dried up to an air-dry condition. Then, a part of the samples prepared in the way was modified: by treatment with a solution of hydrochloric acid $(\mathrm{CHCl}=2 \mathrm{~mol} / \mathrm{dm} 3)$, heat treatment in an oxidizing atmosphere (air) at $250 \pm 1.0^{\circ} \mathrm{C}$ for 5 hours, ozone oxidation for 3 hours. Modified samples, if necessary, were heated at $105 \pm 1.0^{\circ} \mathrm{C}$ to remove residual modifiers, cooled in a desiccator and stored hermetically sealed.

The adsorption of organic substances from aqueous solutions was carried out under the static isothermal conditions $\left(20 \pm 1^{\circ} \mathrm{C}\right)$ in the concentration range $1.0 \cdot 10-4$ to $20 \mathrm{mmol} /$ $\mathrm{dm} 3$, the ratio of the weight of AC: the volume of the test solution is $1: 100$. To establish the equilibrium, the prepared systems were kept with periodic stirring for 24 hours.

The test components concentration was determined by spectrophotometry on a SF-46 instrument (manufactured by LOMO, St. Petersburg) using absolute graduation: phenol and p-chlorophenol by reaction with 4-aminoantipyrine (wavelength $490 \mathrm{~nm}$ ); formaldehyde by reaction with acetylacetone (wavelength $412 \mathrm{~nm}$ ); acetaldehyde by reaction with resorcinol (wavelength $430 \mathrm{~nm}$ ); $\varepsilon$-caprolactam, pyridine and aniline by the self-absorption of solutions in the UV region at a wavelength of 201, 256 and $230 \mathrm{~nm}$, respectively.

Chloroform was determined by vapor phase analysis on a gas chromatograph Kristall2000 (manufactured by the SPA Khromatek, Yoshkar-Ola).

The calibration curves are linear in the optical density range 0-0.90 (R2 $\geq 0.97)$. 


\section{Results}

Investigation of oxygen- and chlorine-containing compounds adsorption

a) adsorption of oxygen- and chlorine-containing compounds (phenol, n-chlorophenol, chloroform, formaldehyde, and acetaldehyde) from aqueous solutions of individual components and their mixtures of AC was studied. According to the obtained experimental data on the adsorption of organic substances from the individual components solutions by different carbon sorbents, adsorption isotherms were constructed as main criteria for evaluating the adsorption properties of the sorbents under the study and made it possible to determine the dependence of the sorbent (sorption capacity) on the adsorbate concentration and nature under equilibrium conditions. Figure 1 shows the adsorption isotherms of the compounds under study from individual aqueous solutions using the example of AG-3 brand.

The theory of monomolecular adsorption (the Freundlich and Langmuir equations), the theory of microspores volume filling (the Dubinin-Radushkevich equation modified for the case of adsorption from an aqueous solution) and Brunauer generalized theory of polymolecular adsorption, Emmett and Teller (BET) were used. The theoretical isotherms of the components adsorption were calculated from the parameters obtained. All adsorption isotherms are analyzed in the corresponding linearization coordinates of these equations and are shown in Fig. 2 using of n-chlorophenol AC AG-3 adsorption.

The calculated values of the adsorption parameters are given in the Table 1 . In the case of acetaldehyde, it is established the use of these equations is not possible to describe the process of its adsorption due to the low value of the correlation coefficients $(\mathrm{R} 2<0.70)$.

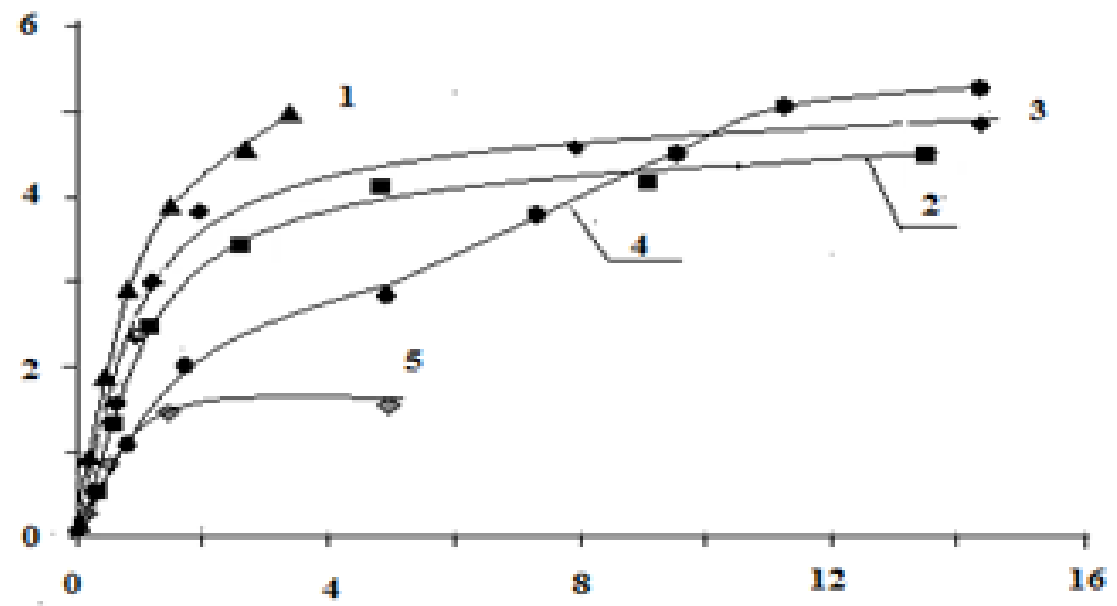

Fig. 1. Adsorption isotherms of oxygen- and chlorine-containing organic substances from individual aqueous solutions with active carbon of AG-3 grade: 1-chloroform; 2-phenol; 3-n-chlorophenol; 4formaldehyde; 5-acetaldehyde 

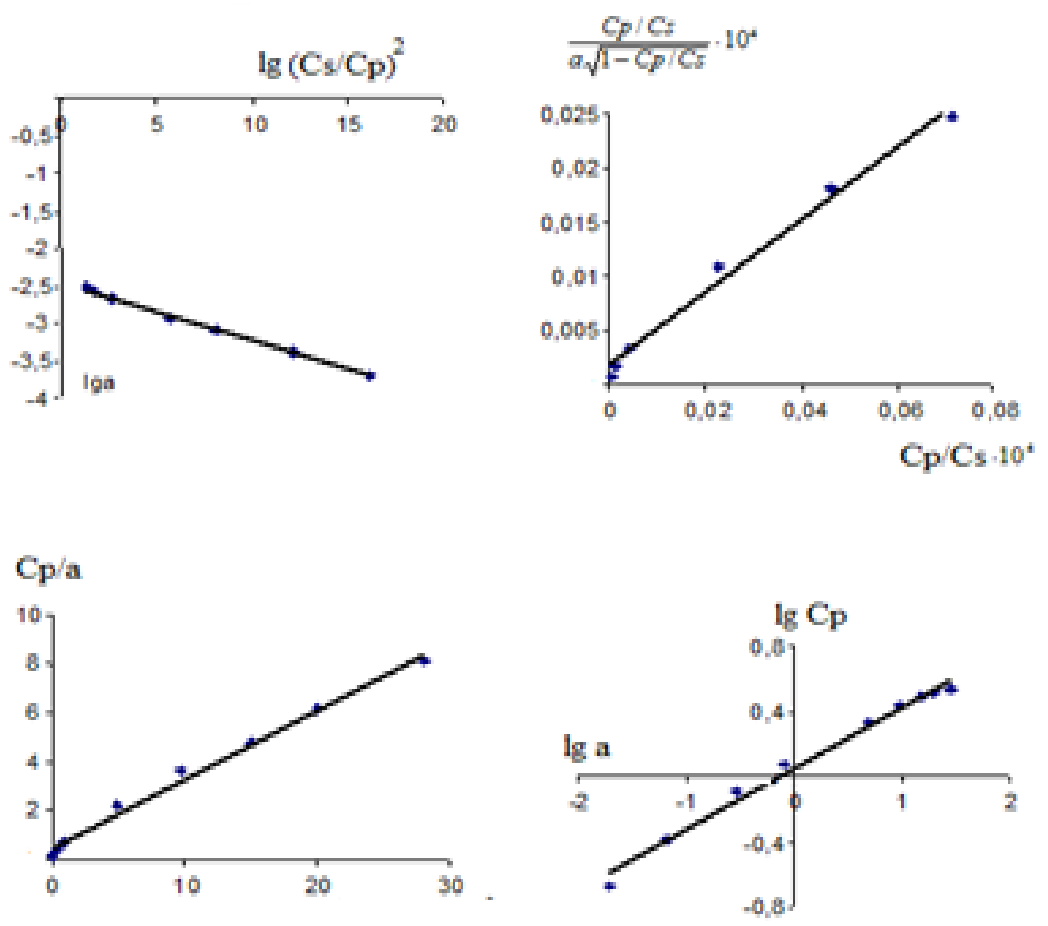

Fig. 2. Isotherms of $n$-chlorophenol adsorption to AC AG-3 in the equations coordinates: a) DubininRadushkevich, b) BET, c) Langmuir, d) Frein-length

Table 1. Parameters of oxygen- and chlorine-containing organic substances adsorption from aqueous solutions by active carbon under the study in the equilibrium conditions

\begin{tabular}{|c|c|c|c|c|c|c|}
\hline \multirow{3}{*}{ Coal grade } & \multicolumn{6}{|c|}{ Type of Equation } \\
\hline & \multicolumn{3}{|c|}{ BET } & \multicolumn{3}{|c|}{ Dubinin - Radushkevich } \\
\hline & $\begin{array}{c}\mathrm{a}_{\max }, \\
\mathrm{mmol} / \mathrm{g}\end{array}$ & $\begin{array}{c}-\mathrm{Q}, \\
\mathrm{kJ} / \mathrm{mol}\end{array}$ & $\mathrm{R}^{2}$ & $\begin{array}{c}\mathrm{a} 0, \\
\mathrm{mmol} / \mathrm{g}\end{array}$ & $\begin{array}{c}\mathrm{W}_{0}, \\
\mathrm{~cm} 3 / \mathrm{g}\end{array}$ & $\mathrm{R}^{2}$ \\
\hline \multicolumn{7}{|c|}{ chloroform } \\
\hline AG-OV-1 & - & - & - & 6.52 & 0.19 & 0.99 \\
\hline SKD-515 & 5.53 & 8.77 & 0.98 & 7.20 & 0.25 & 0.97 \\
\hline AG-3 & 4.60 & 10.10 & 0.97 & 6.80 & 0.11 & 0.98 \\
\hline BAU & - & - & - & 5.63 & 0.32 & 0.96 \\
\hline \multicolumn{7}{|c|}{ phenol } \\
\hline AG-OV-1 & 2.50 & 14.90 & 0.99 & 3.82 & 0.31 & 0.98 \\
\hline SKD-515 & 2.69 & 15.55 & 0.99 & 3.50 & 0.36 & 0.99 \\
\hline AG-3 & 0.89 & 13.45 & 0.98 & 2.14 & 0.25 & 0.97 \\
\hline XAU & 2.01 & 17.42 & 0.99 & 6.75 & 1.12 & 0.99 \\
\hline \multicolumn{7}{|c|}{ n-Chlorophenol } \\
\hline AG-OV-1 & 5.85 & 11.93 & 0.96 & 14.5 & 0.50 & 0.98 \\
\hline SKD-515 & 4.77 & 10.48 & 0.96 & 14.2 & 0.44 & 0.97 \\
\hline AG-3 & 6.15 & 11.98 & 0.97 & 16.1 & 0.52 & 0.98 \\
\hline BAU & 7.07 & 11.84 & 0.95 & 19.2 & 0.68 & 0.94 \\
\hline
\end{tabular}




\begin{tabular}{|l|l|l|l|l|l|l|}
\hline AG-OV-1 & 25.0 & 13.41 & 0.94 & 21.0 & 0.56 & 0.96 \\
\hline SKD-515 & 20.0 & 12.52 & 0.96 & 19.1 & 0.51 & 0.97 \\
\hline AG-3 & 23.8 & 15.91 & 0.98 & 20.1 & 0.54 & 0.97 \\
\hline BAU & 12.8 & 10.83 & 0.93 & 15.6 & 0.42 & 0.95 \\
\hline XAU & 33.3 & 17.34 & 0.97 & 33.1 & 0.33 & 0.98 \\
\hline
\end{tabular}

$\mathrm{a}_{\max }-\max$ is the limiting adsorption capacity of the monolayer, $\mathrm{mol} / \mathrm{g}$; Q - the heat of adsorption, $\mathrm{a}_{0}$ - maximum adsorption; $\mathrm{W}_{0}$ - limiting adsorption volume occupied by the component, $\mathrm{sm} 3 / \mathrm{g}$

As a rule, natural and waste water are complex mixtures of organic substances. In this connection, the adsorption equilibrium on active charcoal in phenol-chloroform, phenol-nchlorophenol, chloroform-n-chlorophenol, formaldehyde-phenol, formaldehydeacetaldehyde systems was compared with the ratio of the components corresponding to their actual content in wastewater and drinking water. A comparison of the phenol and nchlorophenol adsorption isotherms from an aqueous solution of their mixture using the example of AG-3 active coal with adsorption isotherms from their aqueous solutions of individual components is shown in Fig. 3.

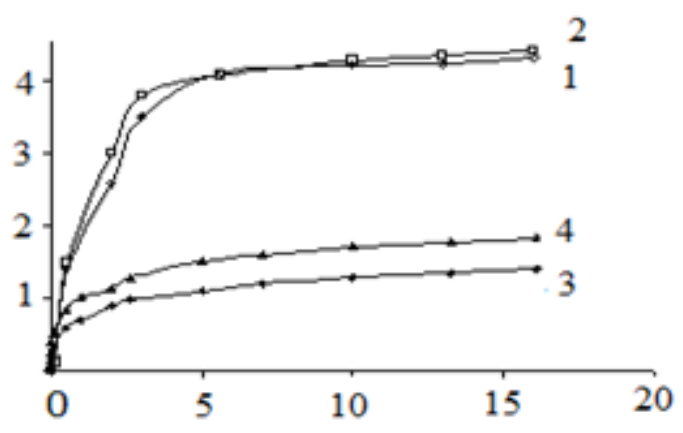

Fig.3. Adsorption isotherms of n-chlorophenol $(1,2)$ and phenol $(3,4)$ on AU AG-3: 2,4-individual component; 1.3 mixture of components

b) Investigation of nitrogen-containing compounds adsorption

The adsorption of nitrogen-containing compounds from individual aqueous solutions under equilibrium conditions was studied for nitrobenzene, $\varepsilon$-caprolactam, aniline and pyridine. The obtained adsorption isotherms in the concentration range under the study (for the example of AC-AG-1 AU) are shown in Fig. 4.

All adsorption isotherms were analyzed in the coordinates of the Langmuir, BET, and Dubinin-Radushkevich equations, modified for the case of adsorption from aqueous solutions and miscible substances. The calculated adsorption parameters are given in Table. 2.

The effect of the second component (phenol) on the adsorption of pyridine was investigated using the example of SKD-515 brand active coal. The obtained adsorption isotherms are shown in the figure. 5 . 


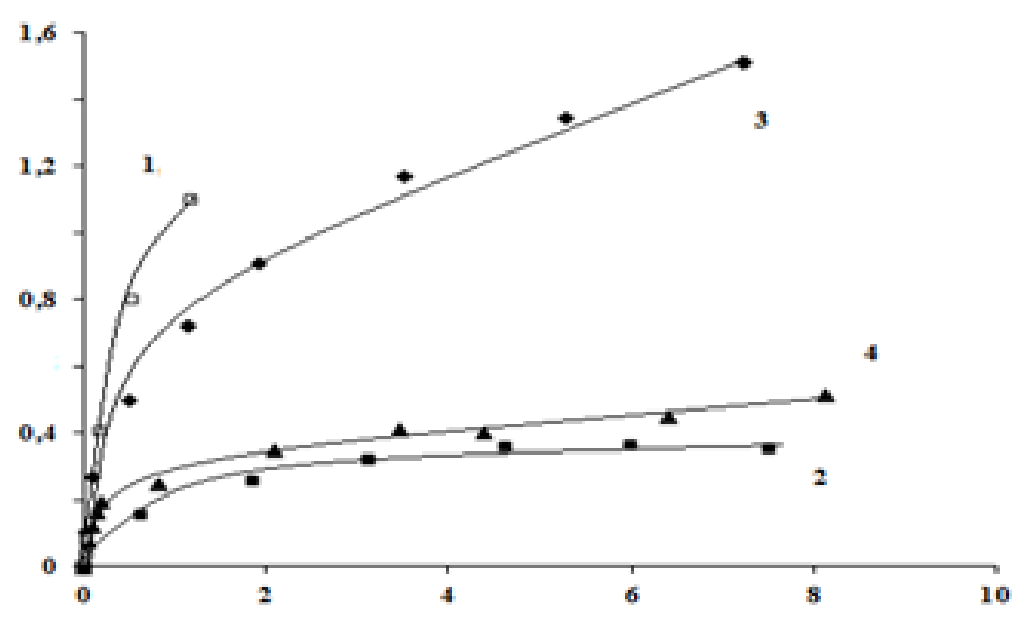

Fig. 4. Adsorption isotherms of nitrogen-containing organic substances from individual aqueous

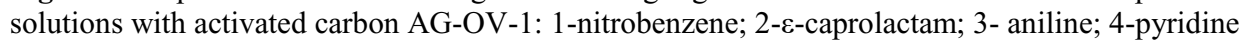

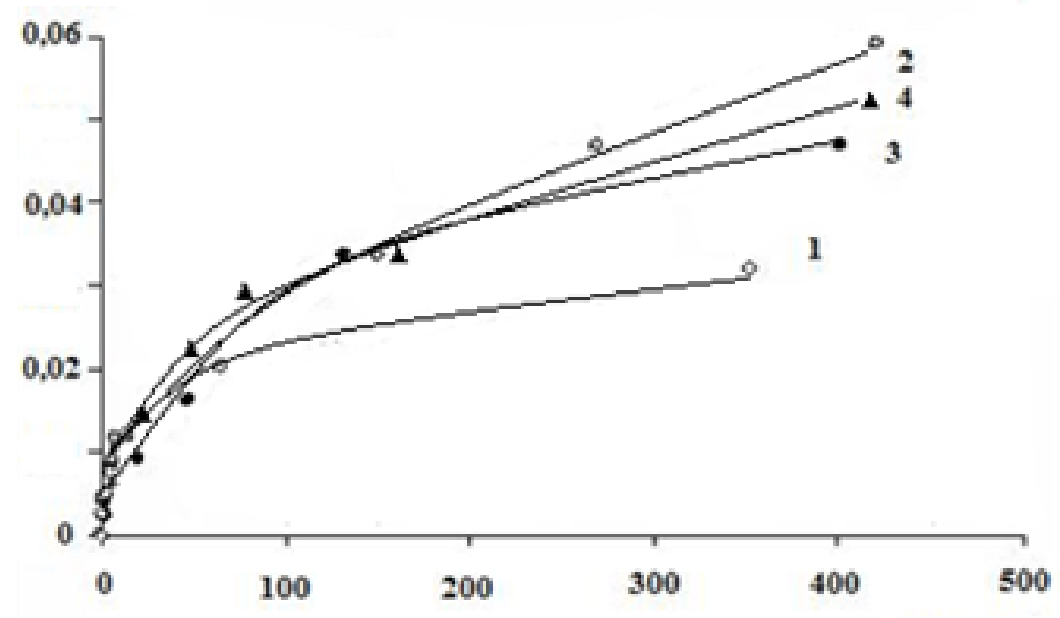

Fig. 5. Adsorption isotherms from aqueous solutions of pyridine with activated carbon of SKD-515 brand: 1 - individual; 2 - from a mixture with phenol at a ratio of 1: 1; 3 - from a mixture with phenol at a ratio of 5: 1; 4- from a mixture with phenol at a ratio of $5: 1$ in the presence of mineral components.

The surface of active carbons is chemically heterogeneous. The oxygen compounds formed on it during the activation increase the adsorption of polar compounds. To further increase the adsorption recovery of such components, the sorbents are further modified. The effect of changes in the state of the surface due to the modification of the nitrogen-containing compounds for adsorption is shown in Fig. 6 
Table 2. Parameters of nitrogen-containing organic substances adsorption from aqueous solutions by active carbon under the study in equilibrium conditions

\begin{tabular}{|c|c|c|c|c|c|c|}
\hline \multirow{3}{*}{ Coal grade } & \multicolumn{6}{|c|}{ Type of Equation } \\
\hline & \multicolumn{3}{|c|}{ Langmuir (BET) } & \multicolumn{3}{|c|}{ Dubinin - Radushkevich } \\
\hline & $\begin{array}{c}\mathrm{G}_{\max }, \\
\mathrm{mmol} / \mathrm{g}\end{array}$ & $\begin{array}{c}-\Delta \mathrm{G}(-\mathrm{Q}), \\
\mathrm{kJ} / \mathrm{mol}\end{array}$ & $\mathrm{R}^{2}$ & $\begin{array}{c}\mathrm{G}_{0}, \\
\mathrm{mmol} / \mathrm{g}\end{array}$ & $\begin{array}{c}\mathrm{W}_{0}, \\
\mathrm{~cm} 3 / \mathrm{g}\end{array}$ & $\mathrm{R}^{2}$ \\
\hline \multicolumn{7}{|c|}{ nitrobenzene } \\
\hline AG-OV-1 & $\begin{array}{c}1.23 \\
(1.19)\end{array}$ & $\begin{array}{c}30.72 \\
(10.27)\end{array}$ & $\begin{array}{c}0.98 \\
(0.99)\end{array}$ & 1.51 & 0.16 & 0.98 \\
\hline SKD-515 & $\begin{array}{c}1.54 \\
(1.52)\end{array}$ & $\begin{array}{c}33.42 \\
(13.47)\end{array}$ & $\begin{array}{c}0.99 \\
(0.98)\end{array}$ & 2.20 & 0.22 & 0.99 \\
\hline ABG & $\begin{array}{c}0.83 \\
(0.82) \\
\end{array}$ & $\begin{array}{c}29.06 \\
(18.66)\end{array}$ & $\begin{array}{c}0.98 \\
(0.98) \\
\end{array}$ & 0.91 & 0.09 & 0.98 \\
\hline \multicolumn{7}{|c|}{$\varepsilon$ - caprolactam } \\
\hline $\mathrm{AG}$ & $\begin{array}{c}0.66 \\
(0.65)\end{array}$ & $\begin{array}{c}- \\
(22.7)\end{array}$ & $\begin{array}{c}0.96 \\
(0.98)\end{array}$ & 2.09 & 0.24 & 0.98 \\
\hline SKD & $\begin{array}{c}0.67 \\
(0.68)\end{array}$ & $\begin{array}{c}- \\
(22.9)\end{array}$ & $\begin{array}{c}0.98 \\
(0.98)\end{array}$ & 2.24 & 0.25 & 0.98 \\
\hline $\mathrm{AG}$ & $\begin{array}{c}0.72 \\
(0.85)\end{array}$ & $(\overline{-})$ & $\begin{array}{c}0.94 \\
(0.96)\end{array}$ & 2.87 & 0.31 & 0.97 \\
\hline \multicolumn{7}{|c|}{ aniline } \\
\hline AG-OV-1 & $\begin{array}{c}1.58 \\
(1.56)\end{array}$ & $\begin{array}{c}28.73 \\
(16.66)\end{array}$ & $\begin{array}{c}0.96 \\
(0.96)\end{array}$ & 2.76 & 0.24 & 0.96 \\
\hline SKD-515 & $\begin{array}{c}0.99 \\
(0.98) \\
\end{array}$ & $\begin{array}{c}28.92 \\
(16.44)\end{array}$ & $\begin{array}{c}0.97 \\
(0.98)\end{array}$ & 1.61 & 0.15 & 0.99 \\
\hline AG-5 & $\begin{array}{c}0.23 \\
(0.23)\end{array}$ & $\begin{array}{c}35.86 \\
(23.75)\end{array}$ & $\begin{array}{c}0.96 \\
(0.97)\end{array}$ & 3.86 & 0.35 & 0.98 \\
\hline ABG & $\begin{array}{c}0.91 \\
(0.89) \\
\end{array}$ & $\begin{array}{c}30.51 \\
(18.46) \\
\end{array}$ & $\begin{array}{c}0.94 \\
(0.96) \\
\end{array}$ & 1.56 & 0.14 & 0.98 \\
\hline \multicolumn{7}{|c|}{ pyridine } \\
\hline AG-OV-1 & 0.50 & 32.78 & 1.00 & 0.89 & 0.07 & 0.97 \\
\hline SKD-515 & 0.42 & 30.29 & 1.00 & 0.83 & 0.06 & 0.98 \\
\hline AG-5 & 0.46 & 30.64 & 1.00 & 1.05 & 0.08 & 0.98 \\
\hline $\mathrm{ABG}$ & 0.66 & 27.22 & 0.97 & 1.42 & 0.11 & 0.94 \\
\hline
\end{tabular}

$\mathrm{G}_{\max }$ - the limiting adsorption capacity of a monolayer, mol/g; $\mathrm{G}$ - Gibbs energy of adsorption, $\mathrm{kJ} / \mathrm{mol}$; $\mathrm{Q}$ - the heat of adsorption, $\mathrm{kJ} / \mathrm{mol} ; \mathrm{G}_{0}$ - the maximum excess adsorption, $\mathrm{mmol} / \mathrm{g} ; \mathrm{W}_{0}$ - the limiting adsorption volume occupied by the component, sm3/g

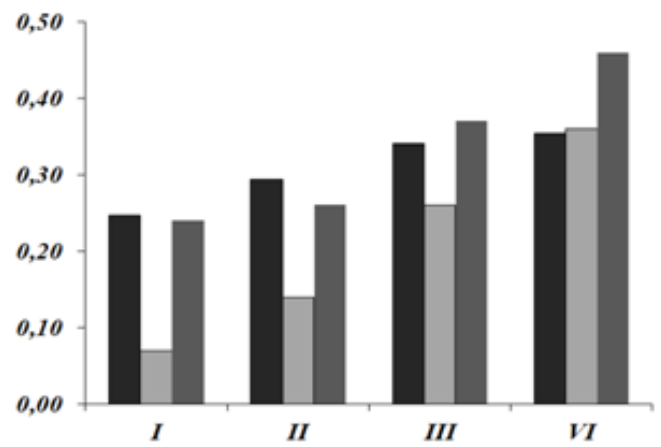

Fig. 6. Maximum adsorption of nitrogen-containing organic substances by modified AG-OV-1 AC samples: I-initial; II-treated with $\mathrm{HCl}$; III - heated at $250^{\circ} \mathrm{C}$; IV - oxidized ozone. 


\section{Discussion}

Investigation of oxygen- and chlorine-containing compounds adsorption

a) analysis of the obtained results of oxygen- and chlorine-containing compounds adsorption made it possible to establish the phenol adsorption from individual aqueous solutions is mainly due to the dispersion interaction $(\mathrm{Q} \leq 14 \mathrm{~kJ} / \mathrm{mol})$, i.e. the contaminant is filled with micropores that are accessible in size. The limiting phenol adsorption investigated by the AU, reduces in the series of KsAC $>$ AG-OV-1> SKD-515> BAC-3.

The introduction of an electronegative substituent (for example, a chlorine atom) into the structure of the phenol molecule increases the component extraction from the aqueous solution. So the chlorophenol adsorption is above the phenol adsorption in $1.5-3.5$ times, depending on active coal brand. This behavior is due to a combination of the following factors: a decrease in solubility due to an increase in size (especially in the process of introducing a substituent into the para- position), a decrease in $\mathrm{pK}$, and in the molecule dipole moment increasing (Fazylova, 2013). The maximum adsorption of the component changes in the series of BAU $>$ AG-3 $\geq$ AG-OV-1 $>$ SKD-515.

Investigation of the equilibrium of both chloroform and formaldehyde adsorption from individual aqueous solutions made it possible to determine that the adsorption of the component proceeds in the micropores. The maximum adsorption of chloroform by the investigated active carbons changes in the series SKD-515> AG-3> AG-OV-1> BAU. The extraction of formaldehyde from aqueous solutions by the investigated AU decreases in the series KsAC $>$ AG-OV-1 $>$ AG-3 $>$ SKD-515> BAC.

The low adsorption of acetaldehyde is due to the fact that it is mixed with water at any ratio. It is thermodynamically more advantageous for it to be in solution than to AU adsorb. A specific interaction predominates within its adsorption. The limiting adsorption capacity of the AU investigated with the respect to acetaldehyde varies in the series: KsAC $>\mathrm{AG}-3>$ SKD-515> AG-OV-1.

Preliminary AU treatment with mineral acids increases the adsorption of phenol by 2 2.5 times, chlorphenol by $80-100 \%$, does not affect formaldehyde adsorption and leads to a decrease in the chloroform adsorption by $1.5-2.5$ times.

Analysis of adsorption behavior in the extraction of chloroform, phenol, $\mathrm{n}$-chlorophenol and acetaldehyde from individual aqueous solutions by one brand of adsorbent showed a good value correlation of limiting adsorption capacity with the substance solubility (Fig. 1). The deviation from this dependence in the high concentrations region of formaldehyde adsorption behavior (the solubility of formaldehyde in water is up to $40 \%$, chloroform is $1 \%$, phenol is $6.7 \%$, $\mathrm{n}$-chlorophenol is $2.7 \%$, acetaldehyde is mixed with water) is probably related to its specific features structure. Formaldehyde is a polar reactive substance. Its interaction with water leads to the formation of mono- and polyhydrates, in addition, it is able to dimerize at high concentrations. Perhaps the formation of formaldehyde dimers in solution and on the surface of active coal causes its high adsorption.

With the simultaneous of phenol and n-chlorophenol adsorption, there is no noticeable effect of phenol presence on the extraction of its chloro derivative. A content of nchlorophenol increase in the mixture by 10 factor leads to a decrease in the limiting adsorption of n-chlorophenol by 5-10\% (Fig. 3).

The study of chloroform and phenol adsorption in a co-presence shows that each of the components is less adsorbed from the mixture than from its individual aqueous solution. The chloroform adsorption decreases to a lesser extent, which is in good agreement with the theoretical propositions that the less soluble component displaces the more soluble one from the surface. In addition, an extra factor may be a smaller size of the chloroform molecule. A mixture of chloroform and n-chlorophenol during adsorption behaves in a similar manner. 
The study of phenol and formaldehyde mixture adsorption from aqueous solutions led to unexpected results. A clearer manifestation of the second stage on the adsorption isotherms of formaldehyde from the mixture in comparison with the individual solution presupposes the formation in the phenol presence of more structured formaldehyde associates with both its own molecules and phenol molecules. The second component introduction in the solution does not affect the phenol adsorption in the entire range of concentrations studied, whereas the formaldehyde adsorption decreases insignificantly.

In the adsorbing process of formaldehyde and acetaldehyde mixture, anomalous behavior is observed. So the adsorption of acetaldehyde does not change within the experimental error, although it is more soluble than formaldehyde (Krasnova, 2015). The isotherms of both components adsorption from the mixture retain the form observed upon adsorption from individual aqueous solutions. Formaldehyde adsorption decreasing, as well as in the case of a mixture with phenol, is observed in the range of concentrations exceeding $80 \mathrm{mg} / \mathrm{dm} 3$ value.

b) Investigation of the nitrogen-containing compounds adsorption

Adsorption of nitrobenzene from individual aqueous solutions is due to the dispersion interaction in available micropores. The maximum adsorption of the component by the investigated active coals changes in the series of SKD-515> AG-OV-1> ABG. Aniline presence slightly reduces nitrobenzene adsorption, but enhances the component molecules interaction with the adsorbent surface (Krasnova, 2011).

In a process of nitrogen-containing substances adsorption with amine / amide group in the structure, a specific interaction with the adsorbents surface with the formation of hydrogen bonds is possible.

In the process of $\varepsilon$-caprolactam adsorption and aniline from individual solutions predominates, the dispersion interaction (L-form of the isotherm according to the Gils classification) with the AC surface is predominant. The possibility of adsorption specific appearance is indirectly confirmed by the high values of adsorption heat, comparable to the hydrogen bond energy. The limiting adsorption capacity of the AC of $\varepsilon$-caprolactam varies in the series of AG-5> SKD-515>AG-OV-1. The presence of $\left(\mathrm{NH}_{4}\right)_{2} \mathrm{SO}_{4}$ in the solution reduces the adsorption of caprolactam at equilibrium concentrations above $5 \mathrm{mmol} / \mathrm{dm} 3$. The maximum aniline adsorption by the investigated AC changes in the series of AG-5> AG-OV-1> SKD-515> ABG. Nitrobenzene presence sharply reduces the aniline adsorption, which is in good agreement with the theoretical propositions.

In the case of pyridine, adsorption due to specific interaction is predominant (H-form of adsorption isotherms according to Gils classification, high value is $\Delta G$ ). The maximum adsorption of the AU component decreases in the series of ABG>AG-5> AG-OV-1> SKD515. In the process of pyridine adsorption from the mixture with phenol, the pyridine recovery is 2.5 times (Fig. 5), in spite of the fact that it is mixed with water at any ratio. The adsorption of pyridine does not alter the introduction of this mineral salt mixture. The growth of pyridine adsorption is associated with a change in the structure of water clusters, as well as the additional interaction of pyridine and phenol molecules on the AC surface due to the formation of hydrogen bonds (Krasnova, 2011).

Unlike oxygen- and chlorine-containing compounds, a good correlation of the adsorption value with the solubility of the nitrogen-containing substance is observed only in the region of high concentrations (at Av> $20 \mathrm{mmol} / \mathrm{dm} 3$ ) (Fig. 4). In the region of filling the monolayer, adsorption is predetermined by a specific interaction, i.e. it depends on the state of adsorbent surface. Any modification leading to an increase (up to a certain value) of oxygen-containing surface compounds leads to an increase in the aniline adsorption, $\varepsilon$ caprolactam and pyridine (Figure 6), which is inversely proportional to the components solubility. 


\section{Conclusions}

A comprehensive study of the adsorption of various classes' organic compounds made it possible to draw the following conclusions. The adsorption of substances interacting with the surface due to the dispersion forces is affected only by the active coal structure. The introduction of mineral substances in this case does not affect the components extraction. The addition of a second organic component to the solution may result in an adsorption decrease, which extent depends on the solubility of both components. The change in the adsorbent surface state can adversely affect the adsorption of such substances.

Adsorption of substances, having groups that are capable of specific interaction in their structure, depends on the AC state surface. Thus, the treatment of active charcoal with acid favorably affects the phenols adsorption, since hydrogen ions adsorbed on the AC surface destroy their dissociation and, consequently, increase adsorption. In the case of nitrogencontaining compounds with amine / amide nitrogen (aniline, $\varepsilon$-caprolactam, and pyridine), the greatest effect is achieved by using oxidative modification leading to an increase in the adsorbent surface of carboxyl and phenolic groups.

In the case of highly soluble, i.e. capable of forming hydrogen bonds with water molecules, a significant role in adsorption also has a change in the solutions structure. The introduction of mineral and/or organic substances capable of strengthening this bond will result in a adsorption decrease, whereas a weakening of the solvent-solute bond will increase adsorption.

The research was carried out within the framework of the State task "Initiative scientific projects" 19.4713.2017 / CU

\section{References}

1. A.M. Koganovskii, N.A. Klimenko, T.M. Levchenko, R.M. Marutovskij, I.G. Purification and use of waste water in industrial water supply, \& Roda, Chemistry. Moscow, (1983)

2. M.P. Kirsanov, The development and application of adsorption processes in purification technologies of natural and waste waters from oxygen-, nitrogen - and chlorine-containing organic compounds, (PhD Thesis), Barnaul (2007)

3. T.A. Krasnova, O.V. Beljaeva, N.S. Golubeva, The treatment of industrial waste waters from nitrogen-containing organic compound, Kemtipp, Kemerovo (2011)

4. A.M. Koganovskii, N.A. Klimenko, T.M. Levchenko \& Roda, I.G. Adsorption of organic substances from water, Chemistry, Leningrad (1990)

5. A.K. Gorelkina, V.V. Kuguk, I.V. Timoshchuk, G.I. Naslednikova, News of higher educational institutions

Construction, http://izvuzstr.sibstrin.ru/uploads/publication/fulltext/N7-2006.pdf (2006)

6. T.A. Krasnova, I.V. Timoshchuk, Ju.S. Shulzhenko, Water and ecology: problems and solutions, 1(61), 3-9 (2015)

7. G.F. Fazylova, E.R. Valinurova, R.M. Khatmullina, F. Kh. Kudasheva, Sorption and chromatographic processes, $\mathbf{5}$ http://www.sorpchrom.vsu.ru/articles/20130524.pdf

(13), 